\title{
Paraserbest Lie Cebirlerinin Metabelyen Çarpımı, 2-Simetrik Kelimeler ve Verbal Altcebir
}

\author{
Zehra VELİOĞLU ${ }^{*}$ iD, Naime EKİCì ${ }^{2}$ \\ ${ }^{1}$ Harran Üniversitesi, Fen-Edebiyat Fakültesi, Matematik Bölümü, Şanlıurfa/Türkiye \\ ${ }^{2}$ Çukurova Üniversitesi, Fen Edebiyat Fakültesi, Matematik Bölümü, Adana/Türkiye
}

Geliş / Received: 23/11/2018, Kabul / Accepted: 13/05/2019

\begin{abstract}
$\ddot{O} \mathbf{z}$
$\mathrm{Bu}$ çalışmada paraserbest Lie cebirlerinin metabelyen çarpımı tanımlanmış ve bu çarpımın paraserbest olduğu gösterilmiştir. F rank1 2 olan bir serbest Lie cebiri ve L sonlu sayıda paraserbest abelyen Lie cebirlerinin metabelyen çarpımı olmak üzere $\mathrm{F}$ de $\mathrm{L}$ tarafından tanımlanan verbal alt cebirin $\mathrm{F}$ " olduğu ispatlanmıştır. Ayrıca bu sonuç ve Fox türevleri kullanılarak L nin bütün 2-simetrik kelimelerinin belirlenebileceği gösterilmiştir.
\end{abstract}

Anahtar Kelimeler: Paraserbest Lie Cebirleri, Metabelyen Çarpım, Simetrik Kelimeler, Verbal Altcebir.

\section{Metabelian Product of Parafree Lie Algebras, 2-Symmetric Words and Verbal Subalgebra}

\begin{abstract}
In this work, the metabelyen product of parafree Lie algebras is defined and it is shown that this product is parafree. Let $\mathrm{F}$ be a free Lie algebra of rank 2 and $\mathrm{L}$ be metabelian product of a finite number of parafree abelyen Lie algebras. It is proved that the verbal subalgebra of L in F equals F". Moreover, by using this result and Fox derivatives, all 2-symmetric words of $\mathrm{L}$ are determined.
\end{abstract}

Keywords: Parafree Lie Algebras, Metabelian Product, Symmetric Words, Verbal Subalgebras.

\section{Giriş}

Baumslag (1967), alt merkezi serisi ile karakterize edilebilen yeni bir grup fikri ortaya atmıştır. Daha sonra Baumslag (1969) bu grupların özelliklerini araştırmış ve bu gruplar1 paraserbest gruplar olarak adlandırmıştır. Baur (1978), paraserbest gruplar için kullanılan kavramları Lie cebirlerine aktarmış ve bu Lie cebirlerinin varlığını kanıtlamıştır. Baumslag'ın ve Baur'un çalışmaları, paraserbest Lie cebirlerinin teorisindeki çalışmalara bir başlan-gıç olmuştur. Paraserbest Lie cebirleri serbest Lie cebirleri ile birçok ortak özelliğinin olmasına rağmen çok çalışılmış bir konu değildir. Bu yüzden paraserbest Lie cebirleri ile ilgili literatürde hala çözülmemiş birçok problem vardır. $\mathrm{Bu}$ durum bu cebir yapısını çalışmamızda motivasyon kaynağı olmuştur.
Pan (2006) bazı aşikar olmayan abelyen grupların metabelyen çarpımı üzerinde çalışmış ve bu çarpımın bütün 2-simetrik kelimelerini belirlemiştir. Grup teorisinde yapılan bu çalışmadan hareketle, bu çalı̧̧mada F rank1 2 olan bir serbest Lie cebiri ve L sonlu saylda paraserbest abelyen Lie cebirlerinin metabelyen çarpımı olmak üzere, $\mathrm{F}$ de $\mathrm{L}$ tarafindan tanımlanan verbal alt cebirin $\mathrm{F}^{\prime \prime}$ olduğu ispatlanmıştır. Ayrıca bu sonuç ve Fox türevleri kullanılarak L nin bütün 2-simetrik kelimelerinin belirlenebi-leceği gösterilmiştir.

\section{Temel Tanım ve Teoremler}

$\mathrm{Bu}$ bölümde çalışmada kullandığımız bazı temel tanım ve teoremlere yer verilmiştir. Ayrıca bu çalı̧̧mada sözü geçen tüm cebirler karakteristiği sıfir olan bir $\mathrm{K}$ cismi üzerinde düşünülecektir. 
Tanım 2.1. $\left(G_{\alpha}\right)_{\alpha \in I}$, K cismi üzerinde tanımlı Lie cebirlerin bir ailesi ve her $\alpha \in I$ için $\left(X_{\alpha} / R_{\alpha}\right), G_{\alpha}$ ailesinin bir sunumu olsun öyle ki $\alpha \neq \beta$ için $X_{\alpha} \cap X_{\beta} \neq \varnothing$ dir.

$$
X=\cup_{\alpha} X_{\alpha} \text { ve } R=\cup_{\alpha} R_{\alpha}
$$

olsun. O halde $G=(X / R)$ sunumuna $\left(G_{\alpha}\right)_{\alpha \in I}$ ailesinin serbest çarpımı denir ve $G=$ $\prod_{\alpha \in I} * G_{\alpha}$ ile gösterilir. Eğer $I=1,2, \ldots, n$ şeklinde sonlu bir küme ise

$$
G=G_{1} * G_{2} * \cdots G_{n}
$$

ile gösterilir. Bu durumda bir $\alpha \in I$ için bir $I_{\alpha}: G_{\alpha} \rightarrow G$ homomorfizmi vardır öyle ki bu homomorfizm $X_{\alpha}$ dan $X$ içine olan birim dönüşümün genişlemesidir.

Tanım 2.2. Bir $L$ Lie cebirinin $\left\{L_{\alpha} \mid \alpha \in I\right\}$ Lie cebirlerinin serbest Lie Çarpımı olması için

$$
\gamma_{1}(L)=L, \gamma_{2}(L)=[\mathrm{L}, \mathrm{L}], \gamma_{3}(L)=\left[\mathrm{L}, \gamma_{2}(L)\right], \ldots, \gamma_{k}(L)=\left[\mathrm{L}, \gamma_{k-1}(L)\right], \ldots
$$

olmak üzere,

$$
L=\gamma_{1}(L) \supset \gamma_{2}(L) \supset \gamma_{3}(L) \supset \ldots \supset \gamma_{k}(L) \supset \ldots
$$

serisine $L$ Lie cebirinin alt merkezi serisi denir. Bu serinin k-yıncı terimi bazen $L_{k}$ ile de gösterilir.

Tanım 2.5. Eğer $\gamma_{k}(L)=\{0\}$ olacak şekilde bir $k$ pozitif tam sayısı varsa $L$ ye nilpotent Lie cebiri denir. Bu $k$ tamsayılarının en küçüğüne ise $L$ nin nilpotentlik sınıfı denir. Eğer L Lie cebirinin nilpotentlik sınıfi 2 ise $\mathrm{L}$ ye bir abelyen Lie cebiridir denir.

Tanım 2.6. $i=1,2, \ldots, k, \ldots$ için $\mathrm{n}_{\mathrm{i}} \geq 1$ olmak üzere pozitif tamsayıların bir $\left\{\mathrm{n}_{1}, \mathrm{n}_{2}, \ldots, \mathrm{n}_{\mathrm{k}, \ldots}\right\}$

$$
L \supseteq L_{n_{1}} \supseteq L_{n_{1}, n_{2}} \supseteq \ldots \supseteq L_{n_{1, \ldots}, n_{i}} \supseteq L_{n_{1}, \ldots, n_{i,}, n_{i+1}} \supseteq \ldots
$$

serisine L Lie cebirinin polisentral serisi denir.

Eğer $n_{1}=n_{2}=2$ ise özel olarak $L_{2,2}=L^{\prime \prime}$ şeklinde gösterilir. Eğer $L^{\prime \prime}=\{0\}$ ise $L$ Lie cebirine metabelyen Lie cebiri denir.

Tanım 2.7. Eğer herhangi bir $0 \neq g C L$ için $L$ Lie cebirinden bir $N$ nilpotent Lie cebirine $\psi_{\mathrm{g}}(\mathrm{g}) \neq 0$ olacak şekilde bir $\psi_{\mathrm{g}}$ homomorfizmi dizisi için $L$ Lie cebirinin polisentral serisi aşağıdaki gibi tanımlanır:

$L_{n_{1}} ; L$ Lie cebirinin alt merkezi serisinin $\mathrm{n}_{1}-$ inci terimi

$L_{n_{1}, n_{2}} ; L_{n_{1}}$ Lie cebirinin alt merkezi serisinin $\mathrm{n}_{2}$-inci terimi

$L_{n_{1, \ldots}, n_{i}, n_{i+1}}=\left(L_{n_{1, \ldots}, \ldots} n_{i}\right)_{n_{i+1}} ; L_{n_{1, \ldots,}, n_{i}}$ nin alt merkezi serisinin $n_{i+1}$-inci terimi olsun. Böylece

varsa $L$ Lie cebirine rezidülü nilpotent denir. $\mathrm{Bu}$ tanıma denk olarak aşağıdaki tanım verilebilir.

$L$ Lie cebirinin alt merkezi serisinin terimlerinin kesişimi sıfır ise $L$ Lie cebirine rezidülü nilpotent Lie cebiri denir. Gerçekten eğer, $\bigcap_{n=1}^{\infty} \gamma_{n}(L)=\{0\}$ ise herhangi bir 
$0 \neq g \in L$ için bir $n$ tamsayısı bulabiliriz öyle ki $\gamma_{n}(L)$ terimi $g$ elemanını içermez.

$\mathrm{L}$ nin alt merkezi serisine $\mathrm{L} / \gamma_{2}(\mathrm{~L})$, $\mathrm{L} / \gamma_{3}(\mathrm{~L}), \ldots$ dizisini karşı1lk getirelim. Bu diziye $\mathrm{L}$ nin alt merkezi dizisi denir. $G, K$ cismi üzerinde bir Lie cebiri olmak üzere eğer $\mathrm{n} \geq 1$ için

$$
\mathrm{L} / \gamma_{n}(\mathrm{~L})=\mathrm{G} / \gamma_{n}(\mathrm{G})
$$

ise $L$ ve $G$ aynı alt merkezi diziye sahiptir denir.

Tanım 2.8. $L, K$ cismi üzerinde tanımlı bir Lie cebiri olsun. Aşağıdaki koşulları sağlaması durumunda $L$ Lie cebirine paraserbest Lie cebiri denir.

i) $\quad L$ rezidülü nilpotenttir,

ii) $\quad F$ bir $X$ kümesi üzerinde serbest Lie cebiri olmak üzere $\phi: F \rightarrow L$ doğal homomorfizmi her $\mathrm{i} \geq 1$ için $\bar{\phi}_{i}: F / \gamma_{i}(F) \rightarrow L / \gamma_{i}(L)$ izomorfizmlerini belirler. Yani $\mathrm{L}$ Lie cebiri bir F serbest Lie cebiri ile aynı alt merkezi diziye sahiptir.
Teorem 2.1. Sonlu çokluktaki sonlu rankl1 paraserbest Lie cebirlerinin serbest çarpımı paraserbesttir.

İspat. İspatı için bakınız (Baur, 1978).

\section{Paraserbest Metabelyen Çarpım}

Tanım 3.1. $P_{1}$ ve $P_{2}$ iki paraserbest Lie cebiri ve $P=P_{1} * P_{2}, P_{1}$ ve $P_{2}$ paraserbest Lie cebirlerinin serbest çarpımı olsun. $\mathrm{D}, \mathrm{P}$ nin kartezyen altcebiri olmak üzere $P_{1}$ ve $P_{2}$ nin metabelyen çarpımı

$$
P_{1} *_{m e t} P_{2}=P / P^{\prime \prime} \cap D
$$

olarak tanımlanır.

Teorem 3.1. $P_{1}$ ve $P_{2}$ iki paraserbest Lie cebiri ve $P=P_{1} * P_{2}$ olsun. Bu durumda $\mathrm{D}, \mathrm{P}$ nin kartezyen alt cebiri olmak üzere $P / P^{\prime \prime} \cap D$ paraserbesttir.

İspat. $P_{1}$ ve $P_{2}$ iki paraserbest Lie cebiri ve $P=P_{1} * P_{2}$ olsun. Bu durumda Teorem 2.1'den $\mathrm{P}$ paraserbesttir. Öncelikle $P / P^{\prime \prime} \cap D$ bölüm cebirinin rezidülü nilpotent olduğunu gösterelim. Bir u $\quad$ $\bigcap_{n=1}^{\infty} \gamma_{n}\left(P / P^{\prime \prime} \cap D\right)$ alalım. Bu durumda her $\mathrm{n}$ için

$X$ kümesine L nin paraüreteç kümesi, $X$ in kümesinin kardinalitesine de $L$ Lie cebirinin rankı denir.

$$
\mathrm{u} \in \gamma_{n}\left(P / P^{\prime \prime} \cap D\right)=\gamma_{n}(\mathrm{P})+\left(P^{\prime \prime} \cap D\right) /\left(P^{\prime \prime} \cap D\right)
$$

olur. $\quad a \in \gamma_{n}(\mathrm{P})+\left(P^{\prime \prime} \cap D\right)$ olmak üzere $u=a+\left(P^{\prime \prime} \cap D\right)$ olsun. Açıktır ki

$$
\bigcap_{n=1}^{\infty} \gamma_{n}\left(P / P^{\prime \prime} \cap D\right)=0
$$

$$
a \in \bigcap_{n=1}^{\infty} \gamma_{n}(\mathrm{P})+\left(P^{\prime \prime} \cap D\right)
$$

dır. $\mathrm{P}$ rezidülü nilpotent olduğundan $a \in$ $\left(P^{\prime \prime} \cap D\right)$ olur. O zaman $u=\left(P^{\prime \prime} \cap D\right)$ olup

dır. O halde $P / P^{\prime \prime} \cap D$ rezidülü nilpotenttir. Şimdi $P / P^{\prime \prime} \cap D$ cebirinin bir serbest Lie cebir ile aynı alt merkezi diziye sahip olduğunu gösterelim.

$$
\left(\gamma_{n}(\mathrm{P})+\left(P^{\prime \prime} \cap D\right)\right) /_{\left(P^{\prime \prime} \cap D\right)} \cong \gamma_{n}(\mathrm{P}) /\left(P^{\prime \prime} \cap D\right)
$$

olduğunu biliniyor. O halde, 


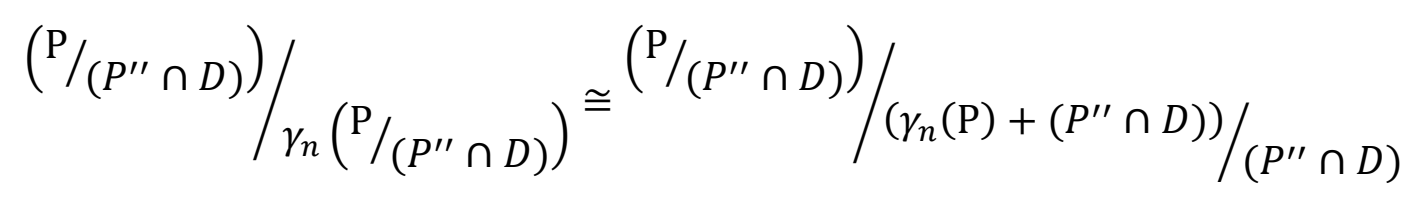

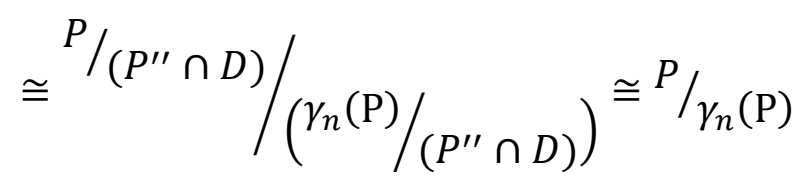

olur. P paraserbest olduğundan

$$
P / \gamma_{n}(\mathrm{P}) \cong \mathrm{F} / \gamma_{n}(\mathrm{~F})
$$

olacak şekilde bir F serbest Lie cebiri vardır. O zaman,

$$
\left.\left(\mathrm{P} / P^{\prime \prime} \cap D\right) /_{\gamma_{n}\left(\mathrm{P} / P^{\prime \prime} \cap D\right.}\right) \cong \mathrm{F} / \gamma_{n}(\mathrm{~F})
$$

dir. Böylece $\mathrm{P} / \mathrm{P}^{\prime \prime} \cap D$ bölüm cebiri paraserbesttir.
Tanım 3.2. $P_{1} *_{m e t} P_{2} \quad$ paraserbest olduğundan bu çarpıma paraserbest metabelyen çarpım da denir.

$\mathrm{F},\{x, y\}$ kümesi tarafından üretilen serbest Lie cebiri ve $L$ herhangi bir Lie cebiri olsun. $F^{\prime} / F^{\prime \prime}, \quad[x, y]$ tarafindan üretilen $U\left(F / F^{\prime \prime}\right)$-modül olup $K[x, y]$ polinom halkasının $F^{\prime} / F^{\prime \prime}$ üzerindeki etkisi

$$
x^{r} y^{s} \cdot[x, y]=[[[x, y] \underbrace{x] \ldots] x]}_{r-\operatorname{def} a} \underbrace{y] \ldots] y]}_{s-\operatorname{def} a}
$$

olarak tanımlanır. Böylece $F^{\prime} / F^{\prime \prime}$ cebirine $[x, y]$ tarafindan üretilen $K[x, y]$ - modül yapısı kazandırılmış olur. O halde her $u \in$ $F^{\prime} / F^{\prime \prime}$ elemanı $\alpha(x, y) \in K[x, y]$ olmak üzere $u=\alpha(x, y)[x, y]$ formundadir.

Tanım 3.3. F de $V=\{u(x, y) \mid u(x, y)=$ $0, \forall g, h \in L\}$ kümesi tarafindan üretilen alt cebire $\mathrm{F}$ de $\mathrm{L}$ tarafindan tanımlanan verbal altcebir denir. Bu cebiri $V(L)$ ile göstereceğiz.

Tanım 3.4. $u(x, y) \in F$ olsun. Eğer $\forall g, h \in$ $L$ için $u(g, h)=u(h, g)$ ise $u(x, y)$, elemanına $\mathrm{L}$ nin $\mathrm{F}$ deki 2-simetrik kelimesi denir.

Not 3.1. $F / V(L)$ rank1 2 olan göreceli serbest (relatively free) Lie cebiri olsun. $u(x, y), \mathrm{L}$ nin $\mathrm{F}$ deki 2-simetrik kelimesi ise $u(x, y) \equiv$ $u(y, x)(\bmod V(L))$ dir. (Fox, 1953).
$F_{n},\left\{x_{1}, \ldots, x_{n}\right\}$ kümesi tarafından üretilen sonlu $\mathrm{n}$ ranklı bir serbest Lie cebiri, $A_{n}=$ $F_{n} / F_{n}^{\prime}$ ve $M_{n}=F_{n} / F_{n}^{\prime \prime}$ olsun. Herhangi bir $v \in F_{n}$ için $\bar{v}$ ve $\tilde{v}$ ile $v$ nin sirasıyla $F_{n} \rightarrow A_{n}$ ve $F_{n} \rightarrow M_{n}$ doğal homomorfizmleri altındaki görüntüleri gösterelim. $i=1,2, \ldots, n$ için $\partial_{i}$ Fox türevi her $\widetilde{u}, \tilde{v} \in U\left(M_{n}\right)$

$\partial_{i}(\tilde{u}+\tilde{v})=\partial_{i}(\tilde{u})+\partial_{i}(\tilde{v})$,

$\partial_{i}\left(x_{j}\right)=\delta_{i j}$,

$\partial_{i}(\tilde{u} . \tilde{v})=\bar{u} \partial_{i}(\tilde{v})+\epsilon(\tilde{v}) \partial_{i}(\tilde{u})$,

olacak şekilde $U\left(M_{n}\right)$ den $U\left(A_{n}\right)$ ye olan lineer bir fonksiyondur. Burada $\delta_{i j}$ Kronecker delta ve $\epsilon: U\left(M_{n}\right) \rightarrow K$ her $i=1, \ldots, n$ için $\epsilon\left(x_{i}\right)=0 \quad$ olacak şekilde bir homomorfizmdir. $\widetilde{w} \in M_{n}$ ve $\bar{\lambda} \in U\left(A_{n}\right)$ ise

$$
\partial_{i}(\bar{\lambda} \widetilde{w})=\bar{\lambda} \partial_{i}(\widetilde{w})
$$

dir (Bryant ve Roman'kov, 1999). Şimdi genelleştirilmiş türevler ve Smel'kin 
gömmesini inceleyelim (Smel'kin ve Syrtsov, 2005).

$A_{1}, \ldots, A_{m}$ sifirdan farkli serbest abelyen Lie cebirleri, $A=A_{1} * \ldots * A_{m}, H=$ $A / A^{\prime}$ ve $G=A / A^{\prime \prime}$ olsun. Her $g \in U(A)$ için

$$
M(H, T)=\left\{\left(\begin{array}{cc}
\bar{a}_{i} & 0 \\
\bar{a}_{i} t_{i} & 0
\end{array}\right) \mid a_{i} \in A_{i}, i=1,2, \ldots, n\right\}
$$

olsun. $i=1,2, \ldots, n$ ve $a_{i} \in A_{i}$ olmak üzere

$$
a_{i} \rightarrow\left(\begin{array}{cc}
\bar{a}_{i} & 0 \\
\bar{a}_{i} t_{i} & 0
\end{array}\right)
$$

olarak tanımlanan fonksiyon çekirdeği $A^{\prime \prime}$ olan bir $\sigma: A \rightarrow M(H, T)$ homomorfizmi belirler. Böylece $A / A^{\prime \prime}, \quad M(H, T)$ içine gömülebilir. Bu gömme Shmel'kin gömmesi olarak bilinir. Herhangi bir $\tilde{a} \in A / A^{\prime \prime}$ için

$$
\sigma(\tilde{a})=\left(\begin{array}{cc}
\bar{a} & 0 \\
\sum_{i=1}^{n} D_{i}(\tilde{a}) t_{i} & 0
\end{array}\right)
$$

olduğu biliniyor. (Burada $i=1,2, \ldots, n$ için $D_{i}$ genelleştirilmiş türevlerdir.)

$$
D_{i}: U(G) \rightarrow U(H)
$$

genelleştirilmiş türevleri aşağıdaki koşulları sağlayan fonksiyonlardır.

1) $D_{i}(\tilde{u}+\tilde{v})=D_{i}(\tilde{u})+D_{i}(\tilde{v}), \quad \tilde{u}, \tilde{v} \in$ $U(G)$

2) $D_{i}(\tilde{u} . \tilde{v})=\bar{u} D_{i}(\tilde{v})+\epsilon(\tilde{v}) \partial_{i}(\tilde{u})$,

3) $D_{i}(\tilde{u})=\bar{u}, \tilde{u} \in A_{i}$ ise

4) $D_{i}(\tilde{u})=0, \tilde{u} \in A_{j}$ ve $i \neq j$ ise

Burada $\epsilon: U(G) \rightarrow K, g \in G$ için $\epsilon(g)=0$ olacak şekilde bir homomorfizmdir. Fox türevlerinde olduğu gibi $\widetilde{w} \in G$ ve $\bar{\lambda} \in U(H)$ ise $D_{i}(\bar{\lambda} \widetilde{w})=\bar{\lambda} D_{i}(\widetilde{w})$ eşitliği geçerlidir.

Şimdi bu çalışmamızın temel sonuçlarını vereceğiz.

\section{Verbal Altcebir ve 2-Simetrik Kelimeler}

$P_{1}, \ldots, P_{n}$ paraserbest abelyen Lie cebirleri ve $P=P_{1} * \ldots * P_{n}$ ise $P_{1}, \ldots, P_{n}$ paraserbest Lie cebirlerinin serbest çarpımı olsun.

Teorem 4.1. $L=P / P^{\prime \prime}$ ise $\mathrm{F}$ de $\mathrm{L}$ tarafindan tanımlanan verbal altcebiri $F^{\prime \prime}$ ne eşittir. Yani $V(L)=F^{\prime \prime}$ dür.

İspat. $F^{\prime \prime} \subset V(L)$ olduğu açıktır. Şimdi $V(L) \subset F^{\prime \prime}$ olduğunu gösterelim. $u(x, y) \in$ $V(L)$ olsun. $F^{\prime}\left(\bmod F^{\prime \prime}\right),[x, y]$ tarafindan üretilen $U\left(F / F^{\prime}\right)$ - modül olduğundan her $w \in$ $F^{\prime}\left(\bmod F^{\prime \prime}\right)$ eleman1, $\alpha(x, y) \in U\left(F / F^{\prime}\right)$ olmak üzere

$$
w \equiv \alpha(\bar{x}, \bar{y})[x, y]\left(\bmod F^{\prime \prime}\right)
$$

formundadir. $\mathrm{Bu}$ durumda

$u(x, y) \equiv a x+b y+\alpha(\bar{x}, \bar{y})[x, y]\left(\bmod F^{\prime \prime}\right)$

olduğunu kabul edebiliriz. Her $\tilde{v}_{1}, \tilde{v}_{2} \in L$ için $u\left(\tilde{v}_{1}, \tilde{v}_{2}\right)=0$ olduğu tanımdan dolayı açıktır. Şimdi $\tilde{v}_{1}=\tilde{c}_{1} \neq 0$ ve $\tilde{v}_{2}=0$ alalım. $\mathrm{O}$ zaman

$$
u\left(\tilde{c}_{1}, 0\right)=a \cdot c_{1}=0
$$

ve dolayısıyla $a=0$ elde edilir. Benzer şekilde $\tilde{v}_{1}=0$ ve $\tilde{v}_{2}=\tilde{c}_{2} \neq 0$ seçilirse

$$
u\left(0, \tilde{c}_{2}\right)=b \cdot c_{2}=0
$$

ve $b=0$ bulunur. Buradan

$$
u(x, y) \equiv \alpha(\bar{x}, \bar{y})[x, y]\left(\bmod F^{\prime \prime}\right)
$$


yani $u(x, y) \in F^{\prime}$ bulunur. Şimdi $v_{1}=\tilde{c}_{1} \in$ $P_{1}, v_{2}=\tilde{c}_{2} \in P_{2}$ alalım. $\tilde{c}_{1} \neq 0, \tilde{c}_{2} \neq 0$ dir. $\mathrm{O}$ zaman

$$
u\left(\tilde{c}_{1}, \tilde{c}_{2}\right)=\alpha\left(\tilde{c}_{1}, \tilde{c}_{2}\right)\left[\tilde{c}_{1}, \tilde{c}_{2}\right]=0
$$

olur. $\tilde{c}_{1} \mathrm{e}$ göre genelleştirilmiş türevini alalım. Türevin değeri $U\left(F / F^{\prime}\right)$ de olup

$$
-\alpha\left(\bar{c}_{1}, \overline{c_{2}}\right) \overline{c_{2}} \overline{c_{1}}=0
$$

buluruz. $U\left(F / F^{\prime}\right)$ tamlık bölgesi olduğundan $\alpha\left(\bar{c}_{1}, \bar{c}_{2}\right)=0$ elde edilir. Her $\tilde{c}_{i} \in P_{i}$ ve $\tilde{c}_{j} \in$ $P_{j}$ için $\alpha\left(\tilde{c}_{i}, \tilde{c}_{j}\right)=0$ olup $\alpha(x, y)=0$ dir. Bu durumda $u(x, y) \in F^{\prime \prime}$ olup $V(L)=F^{\prime \prime}$ dür.

$$
u(x, y)=\lambda x+\lambda y+\alpha(x, y)[x, y]\left(\bmod F^{\prime \prime}\right)
$$

formundadir. Burada $\alpha(x, y) \in U\left(F / F^{\prime}\right)$ ve $\alpha(x, y)=-\alpha(y, x)$ dir.

İspat. $u(x, y), \mathrm{M}$ nin $\mathrm{F}$ deki 2-simetrik bir kelimesi ise $u(x, y)=u(y, x)$ dir. $F^{\prime} / F^{\prime \prime}$, $U\left(F / F^{\prime}\right)$-modül olduğundan $u(x, y)$ elemanı, $v \in F^{\prime \prime}$ ve $\alpha(x, y) \in U\left(F / F^{\prime}\right)$ olmak üzere

$$
u(x, y)=a x+b y+\alpha(x, y)[x, y]+v
$$

şeklinde yazılabilir. $\tilde{x}=x+F^{\prime \prime}, \tilde{y}=y+F^{\prime \prime}$ olduğu göz önüne alınırsa

$$
u(\tilde{x}, \tilde{y}) \equiv a \tilde{x}+b \tilde{y}+\alpha(\tilde{x}, \tilde{y})[\tilde{x}, \tilde{y}]
$$

olduğu açıktır. $u(\tilde{x}, \tilde{y})=u(\tilde{y}, \tilde{x})$ eşitliği göz önüne alalım. Buradan

$$
\begin{aligned}
& a \tilde{x}+b \tilde{y}+\alpha(\tilde{x}, \tilde{y})[\tilde{x}, \tilde{y}] \\
& \quad=a \tilde{y}+b \tilde{x}+\alpha(\tilde{y}, \tilde{x})[\tilde{y}, \tilde{x}]
\end{aligned}
$$

ve

$$
\begin{gathered}
a \tilde{x}+b \tilde{y}=a \tilde{y}+b \tilde{x} \\
(\alpha(\tilde{x}, \tilde{y})+\alpha(\tilde{y}, \tilde{x}))[\tilde{x}, \tilde{y}] \equiv 0
\end{gathered}
$$

elde edilir. (1) eşitliğinden $a=b$ bulunur. (2) eşitliğinin $\tilde{x}$ e göre genelleştirilmiş türevini alalım.

$$
-(\alpha(\bar{x}, \bar{y})+\alpha(\bar{y}, \bar{x})) \bar{y} \cdot \bar{x}=0,
$$

formundadir. $u(x, y)=u(y, x)$ olup
Sonuç 4.1. $\mathrm{M}, P_{1}, \ldots, P_{n}$ paraserbest abelyen Lie cebirlerinin metabelyen çarpımı ise $V(M)=F^{\prime \prime}$ dür.

İspat. $M=P / P^{\prime \prime} \cap D$ olup $i=1,2, \ldots, n$ için $P_{i}$ ler abelyen olduğundan $P^{\prime \prime} \cap D=P^{\prime \prime}$ dür. Dolayisiyla $M=P / P^{\prime \prime}$ olur. Teoremden $V(M)=F^{\prime \prime}$ olduğu görülür.

Şimdi 2-simetrik bir kelimelerin $V(M)$ tarafından nasıl belirlendiğini gösterelim.

Teorem 4.2. $u(x, y), \mathrm{M}$ nin $\mathrm{F}$ deki 2-simetrik bir kelimesi olsun. O zaman $u(x, y)$,

bulunur. $U\left(F / F^{\prime}\right)$ bir tamlık bölgesi olduğundan $\alpha(\bar{x}, \bar{y})+\alpha(\bar{y}, \bar{x})=0$ bulunur. $a=b=\lambda$ diyelim. O halde $u(x, y)$ elemanı

$u(x, y)=\lambda x+\lambda y+\alpha(x, y)[x, y]\left(\bmod F^{\prime \prime}\right)$

$$
\alpha(x, y)=-\alpha(y, x)
$$

Sonuç 4.2. $u(x, y), \mathrm{M}$ nin $\mathrm{F}$ deki 2-simetrik kelimesi ise $u(x, y)-u(y, x) \in V(M)$ dir.

İspat. $u(x, y), \mathrm{M}$ nin $\mathrm{F}$ deki 2-simetrik kelimesi olsun. Teoremden $u(x, y)$

$$
\begin{gathered}
u(x, y)=\lambda x+\lambda y+\alpha(x, y)[x, y]+v, \\
\alpha(x, y) \in U\left(F / F^{\prime}\right), v \in F^{\prime \prime}
\end{gathered}
$$

formundadır. $u(x, y), 2$-simetrik olduğundan

$$
u(x, y)-u(y, x)=v(x, y)-v(y, x)=0
$$

elde edilir. O halde her $g, h \in M$ için

$$
u(g, h)-u(h, g)=v(g, h)-v(h, g)=0
$$

dir. Yani $u(x, y)-u(y, x) \in V(M)$ dir.

$w \in V(M)$ ise her $g, h \in M$ için $w(g, h)=0$ olup $w(g, h)=w(h, g)$ dir. Yani $w(x, y) \in$ $F^{\prime \prime}, \mathrm{M}$ nin $\mathrm{F}$ deki 2-simetrik bir kelimesidir. Diğer taraftan 


$$
\begin{gathered}
u(x, y)=a x+b y+ \\
\alpha(x, y)[x, y]\left(\bmod F^{\prime \prime}\right), \\
\alpha(x, y) \in U\left(F / F^{\prime}\right) \text { ve } \alpha(x, y)=-\alpha(y, x),
\end{gathered}
$$

ise $w \in F^{\prime \prime}$ herhangi bir eleman olmak üzere

$$
u(x, y)=a x+a y+\alpha(x, y)[x, y]+w
$$$$
u(x, y)=x+y+\alpha(x, y)[x, y]+w, w \in F^{\prime \prime},
$$

$\mathrm{M} \operatorname{nin} \mathrm{F}$ deki 2-simetrik kelimesidir.

Gerçekten

$$
\begin{gathered}
u(x, y)=x+y+\left(x^{2} y-y^{2} x\right)[x, y]\left(\bmod F^{\prime \prime}\right) \\
=x+y+[[[[x, y], x], x], y]-[[[[x, y], y], y], x]\left(\bmod F^{\prime \prime}\right)
\end{gathered}
$$

ve

$$
u(y, x)=y+x+[[[[y, x], y], y], x]-[[[[y, x], x], x], y]\left(\bmod F^{\prime \prime}\right)
$$

olup $u(x, y)=u(y, x)$ dir.

\section{Açıklamalar}

$\mathrm{Bu}$ çalışma 31. Ulusal Matematik Sempozyumunda (UMS 2018) sunulmuştur.

\section{Kaynaklar}

Baumslag, G. 1967. ' 'Groups with the same lower central sequence as a relatively free group I. The groups', Trans. Amer. Math. Soc., 129, 308-321.

Baumslag, G. 1969. 'Groups with the same lower central sequence as a relatively free group. II Properties", Trans. Amer. Math. Soc., 142, 507-538.

Baur, H. (1978). ' 'Parafreie Liealgebren und homologie'", Diss. Eth Nr., Zurich, 6126, 60p.

Bryant, R.M. and Roman'kov, V.A. 1999. "'Automorphism groups of relatively free groups", Math. Proc. Camb. Phil. soc., 127(3), 411-424.
Fox, R.H. 1953. 'Free differential calculus. I. Derivations in the free group ring', Ann. Math. 57(3), 547-560.

Pan, J. 2006. 'On 2-Symmetric words and verbal subgroup of metabelian product of abelian groups', Alg. Colloquium, 13(3), 535-540.

Shmel'kin, A.L. and Syrtsov, A.V. 2005. ' On embeddings of some factor algebras of free sums of Lie algebras', J. Math. Sci. 13( 6), 6148-6152. 